\title{
Experimental Study of Plasma Breakdown by Toroidally Injected Electron Cyclotron Waves in Heliotron J
}

\author{
Bo LU, Kazunobu NAGASAKI ${ }^{1)}$, Tohru MIZUUCHI ${ }^{1)}$, Hiroyuki OKADA ${ }^{1)}$, Takashi MINAMI ${ }^{1)}$, \\ Shinji KOBAYASHI ${ }^{1)}$, Satoshi YAMAMOTO ${ }^{1)}$, Shinsuke OHSHIMA ${ }^{1)}$, Shigeru KONOSHIMA ${ }^{1)}$ \\ and Fumimichi SANO ${ }^{1)}$ \\ Center for Fusion Science, Southwestern Institute of Physics, P.O.Box 432, Chengdu, Sichuan, 610041, China \\ ${ }^{1)}$ Institute of Advanced Energy, Kyoto University, Gokasho, Uji, Kyoto 611-0011, Japan
}

(Received 22 May 2012 / Accepted 5 October 2012)

\begin{abstract}
Plasma breakdown using electron cyclotron waves with toroidally inclined launch has been studied experimentally in the stellarator/heliotron device, Heliotron J. The experimental results show that the second harmonic $\mathrm{X}$-mode breakdown has a weak dependence on the parallel refractive index, $N_{\|}$, for the small toroidal injection angle range. When the fundamental resonance exists inside the vacuum chamber, the breakdown occurs quickly, independent of the toroidal injection angle.
\end{abstract}

(C) 2012 The Japan Society of Plasma Science and Nuclear Fusion Research

Keywords: breakdown, ECH, stellarator/heliotron, Heliotron J

DOI: $10.1585 /$ pfr.7.1202153

High-power electron cyclotron waves (ECW) are widely utilized for plasma production in toroidal magnetic fusion devices. In superconductive tokamaks such as International Thermonuclear Experimental Reactor (ITER), the superconducting solenoid and the thick vessel walls limit the available toroidal electrical field for plasma breakdown well below the values used in most of the operating tokamaks. Since pre-ionization using ECW is effective for plasma start-up at low toroidal electrical field in many tokamaks [1], assisted plasma breakdown is highly desirable for ITER to increase the operational margin. In stellarator/heliotron devices, on the other hand, high power ECW of fundamental and second harmonic resonance frequencies are routinely used for reliable plasma start-up [2].

Several theoretical and experimental works have been carried on the plasma breakdown in tokamaks [3-7] and stellarator/heliotron devices [8,9]. Fundamental plasma breakdown can be explained simply by a linear theory, while second-harmonic plasma breakdown requires consideration of nonlinear wave particle interactions, since the linear energy increment of seed electrons is proportional to the gyroratius squared, which is practically zero at the initial phase $[2,8]$. Both the fundamental and second harmonic breakdowns are experimental studied; the injected power, polarization, poloidal injection angle, prefill pressure, magnetic configuration are scanned in several machines. Most of these studies were performed with ECW launched perpendicular to the magnetic field. Since oblique ECW launch with the toroidal angle of $20 \mathrm{deg}$ is planned in ITER, it is an issue to clarify the toroidal angle dependence. In order to understand the physics of the plasma breakdown using the toroidally injected ECW, we have experimentally studied plasma breakdown in the helical-axis heliotron device, Heliotron $\mathrm{J}$.

The Heliotron $\mathrm{J}$ device is a medium sized helical device with major radius, $R=1.2 \mathrm{~m}$, averaged plasma minor radius, $a=0.1-0.2 \mathrm{~m}$, low-shear rotational transform $\iota / 2 \pi=0.3-0.8$ and magnetic well depth $1.5 \%$, at the plasma edge [10]. The magnetic field is formed by combining an $L / M=1 / 4$ helical coil, two kinds of toroidal coils and inner and vertical coils. The plasma operation regimes are widely extended by virtue of its configuration flexibility. The magnetic configuration is scanned by varying the current ratios in each coil in order to investigate the properties of the breakdown in some magnetic configurations. Two configurations are involved in this study: medium bumpiness with $\varepsilon_{b}=0.06$ and high bumpiness with $\varepsilon_{b}=0.15$, where $\varepsilon_{b}$ is the bumpiness of the Fourier component in the magnetic field. The magnetic field strength is set so as to locate the second harmonic resonance at plasma central region.

A $70 \mathrm{GHz}$ ECH system is used for studying the plasma breakdown in Heliotron J. The detail of the $70 \mathrm{GHz} \mathrm{ECH}$ system is described in [11]. The injected power is kept constant at about $300 \mathrm{~kW}$ and the pulse duration is $120 \mathrm{~ms}$ in the experiment reported here. The ECW is launched with a focused Gaussian beam. A lower power test shows that the beam radius of $1 / e^{2}$ power is $3 \mathrm{~cm}$ at magnetic axis, smaller than the minor radius. The injection angle can be widely controlled both toroidally and poloidally by steering a mirror in the launcher. The available parallel refractive index of launched ECW is $0<N_{\|}<0.6$. In toroidal scan, the poloidal angle is adjusted such that the 


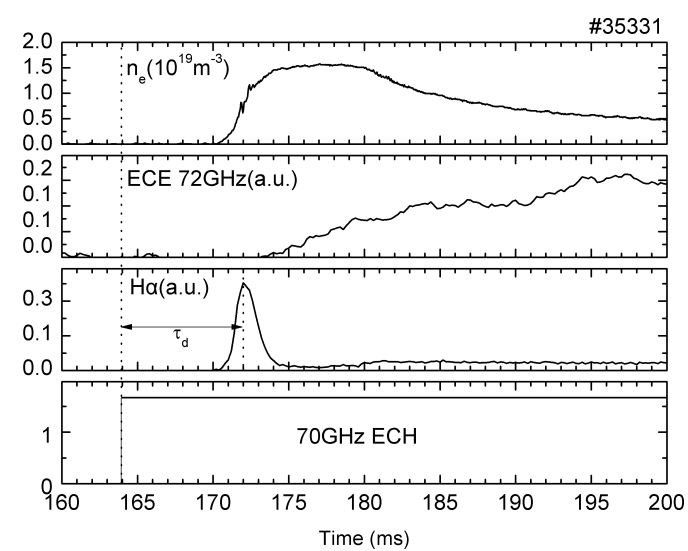

Fig. 1 Time evolution of the plasma breakdown. The line averaged electron density, ECE signal, $\mathrm{H}_{\alpha}$ intensity and $\mathrm{ECH}$ pulse are plotted.

injected beam crosses the magnetic axis. The polarization is configured in the X-mode by a linear polarizer at the perpendicular case. In the oblique injection experiment, the polarization is kept the same as in the perpendicular injection case, so the polarization, especially the ellipticity, is not fully optimized in this study. However, the X-mode fraction is relatively large, more than $80 \%$ for $0<N_{\|}<$ 0.6 .

Diagnostics such as a microwave interferometer, $\mathrm{H}_{\alpha}$ measurement are used to observe the initial phase of the plasma discharge. The working gas is $\mathrm{D}_{2}$. The gas pressure is of the order of $10^{-5}$ Torr, which is controlled by gas puffing. The gas puffing condition is almost fixed in this experiment. The wall conditioning is performed by titanium gettering.

A typical time evolution of an ECH plasma breakdown is shown in Fig. 1. A few milliseconds after the ECH is switched on, the $\mathrm{H}_{\alpha}$ signal starts to rise up. And then the line averaged electron density signal shows a slow increase. Here the interferometer views the central region vertically at the straight section. The $\mathrm{H}_{\alpha}$ has a peak before the electron density is saturated. The time delay of an initial $\mathrm{H}_{\alpha}$ peak and its peak intensity, caused by the ionization process after the ECH turn-on is estimated as a parameter of breakdown characteristics. The time delay is defined as the time between the beginning of an $\mathrm{ECH}$ pulse and the peak $\mathrm{H}_{\alpha}$ time, the same as in most papers.

Figure 2 shows the time delay of the $\mathrm{H}_{\alpha}$ signal and calculated peak power density of injected Gaussian beam as a function of $N_{\|}$in the medium-bumpiness configuration. The peak power density is calculated at the magnetic axis which is close to the resonant layer and the effect of the X-mode fraction is included. $N_{\|}$is scanned from 0 to 0.5 , corresponding to the change in toroidal injection from 0 to $30 \mathrm{deg}$. The fundamental resonance is out of the vacuum vessel and the second harmonic resonance lies near the magnetic axis in this configuration. It can be seen that

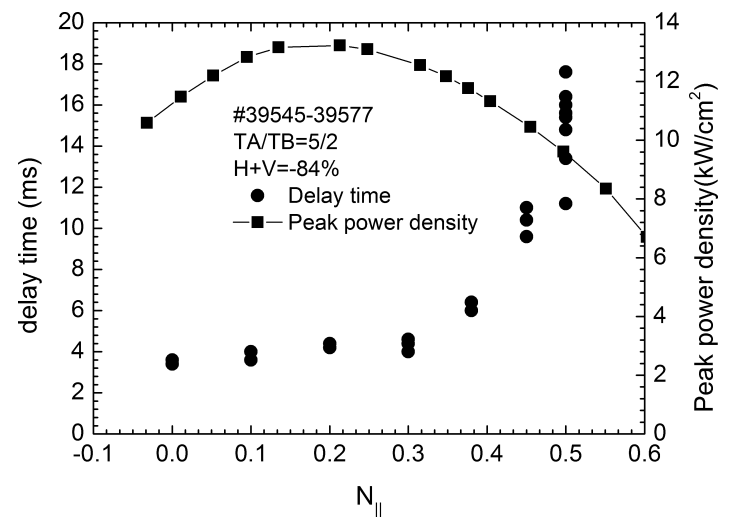

Fig. $2 N_{\|}$dependence of the plasma breakdown in mediumbumpiness configuration. The second harmonic EC resonance layer is located at magnetic axis.

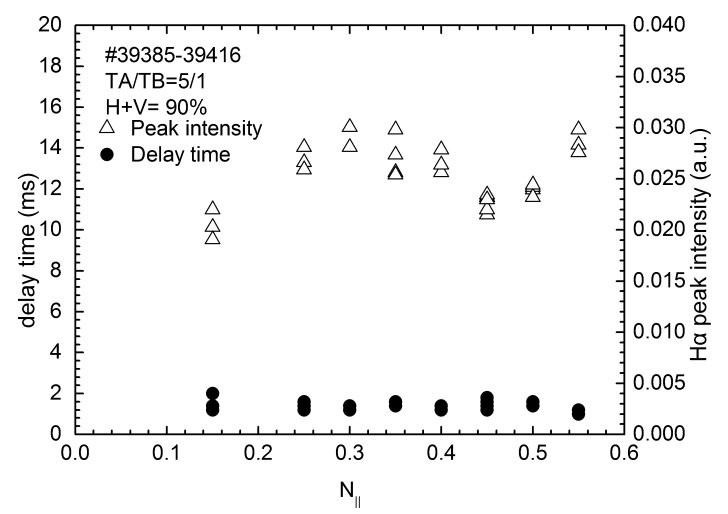

Fig. $3 N_{\|}$dependence of plasma breakdown in high-bumpiness configuration. The second harmonic $\mathrm{EC}$ resonance layer is located at magnetic axis, and the fundamental EC resoancne layer is located at the plasma edge.

the time delay is weakly dependent on the $N_{\|}$at $N_{\|}<0.4$, and the time delay increases quickly at $N_{\|}>0.4$. The parallel refractive index, $N_{\|}=0.4$, corresponds to the toroidal injection angle of $24 \mathrm{deg}$. This $N_{\|}$dependence is similar to an experimental result in TJ-II [1]. These suggest that second harmonic plasma breakdown is less affected in the small toroidal angle launch if the launched power is larger than a critical threshold. For larger $N_{\|}$, the peak power density of the injected Gaussian beam at the resonance layer decreases, but it is similar to the power density at $N_{\|}=0.0$. Since the delay time is not a simple monotonic function of the peak power density, another unknown effect should also be included to explaining the $N_{\|}$dependence of delay time, particularly at large $N_{\|}$. This study is left for future.

Figure 3 shows the dependence of the time delay and intensity of $\mathrm{H}_{\alpha}$ signal on $N_{\|}$at the high-bumpiness configuration. In contrast to the medium-configuration case, the breakdown occurs quickly after the ECH turn-on and has a very weak $N_{\|}$dependence in this configuration. What is different from the medium-bumpiness configuration is 
that both the fundamental $B=2.5 \mathrm{~T}$ resonance and second harmonic $B=1.25 \mathrm{~T}$ resonance are located inside the vacuum chamber. The fundamental X-mode from the lowfield side cannot access the resonance due to evanescent layer as the electron density rises, while that from the highfield side may contribute to plasma breakdown. Since the multi-reflected waves have a random polarization, part of the reflective waves is possibly converted into the O-mode, contributing to the fundamental breakdown. A CCD camera measurement confirmed that the total light started to appear first at the plasma edge where the fundamental resonance layer was located.

The authors are grateful to the Heliotron $\mathbf{J}$ staff for conducting the experiments. One of the authors (K.N.) thanks E. Ascasibar for useful discussion. This work was performed with the support and under the auspices of the JSPS Asian CORE Program, the Collaboration Program of the Laboratory for Complex Energy Processes, IAE, Kyoto
University, and the NIFS Collaborative Research Program (NIFS08KOAR010, NIFS10KUHL030), the NIFS/NINS project of Formation of International Network for Scientific Collaborations, and a Grant-in-Aid for Scientific Research, MEXT.

[1] J. Stober et al., Nucl. Fusion 51, 083031 (2011).

[2] K. Nagasaki et al., J. Korean Phys. Soc. 49, S18 (2006).

[3] A.C.C. Sips et al., Nucl. Fusion 49, 085015 (2009).

[4] B. Lloyd et al., Nucl. Fusion 31, 2031 (1991).

[5] G.L. Jackson et al., Phys. Plasma 17, 056116 (2010).

[6] G. Granucci et al., Nucl. Fusion 51, 073042 (2010).

[7] Y.S. Bae et al., Nucl. Fusion 49, 022001 (2009).

[8] A. Cappa et al., Nucl. Fusion 41, 363 (2001).

[9] K. Nagasaki et al., Nucl. Fusion 45, 13 (2005).

[10] T. Obiki et al., Nucl Fusion 41, 833 (2001).

[11] K. Nagasaki et al., Contrib. Plasma Phys. 50, 656 (2010). 